International Journal of Engineering \& Technology, 7 (2.20) (2018) 83-85
International Journal of Engineering \& Technology
SPC
Website: www.sciencepubco.com/index.php/IJET
Research paper

\title{
A fabric architecture towards block chain application using hyper ledger
}

\author{
Md. Haseeb*, K. Raja Sekhar, Y.V. Spandana, M. Syam \\ Department of Computer Science and Engineering, Koneru Lakshmaiah Educational Foundation, Vaddeswaram, Andhra Pradesh, India. \\ *Corresponding author E-mail: mohammadhaseeb514@gmail.com
}

\begin{abstract}
Block chain is a trust which can be best understood by the state machine replication, It is decentralized distributed ledger which is along all peers in the network connected through nodes over Internet. Every Node in the chain have equal stake and also the main factor is every node which have greater CPU cycles has a chance to operate node by spending those CPU cycles and also show Proof Of Work. Hence when the block chain is used in Business Models it loses its Private transactions and Confidential Contracts.
\end{abstract}

Keywords: Block chain, Hyper ledger, fabric, transaction, security.

\section{Introduction}

Bitcoin and its block chain have permitted commonly doubting elements to perform monetary installments without depending on a focal trusted outsider while offering a straightforward and uprightness secured information stock-piling. Because of these properties, Block chain as an innovation has increased much consideration past the reason of money related exchangesconveyed distributed storage, shrewd property, web of things, production network administration social insurance, possession and sovereignty dissemination, and decentralized self-ruling associations just to give some examples. In opposition to Bitcoin's permission less Block chain, where any essayist can join whenever, alleged permissioned block chains have been proposed, where just an approved arrangement of substances is permitted to compose \& perused the individual block chain. A permissioned block chain, in any case, shares similitude's with an incorporated database, and this normally raises the inquiry whether a block chain is more qualified than a unified database

Block chain applications:

\section{i) Financial services}

1. Insurance: The insurance claiming processing is so frustrating. Processors need to swim through false claims, divided information sources, or relinquished approaches for clients to express a couple of -and process these structures physically. Space for mistake is enormous. The block chain gives an ideal structure to hazard free administration and directness. Its encryption properties let Safety net providers to snap the duty to be protected.

2. Payments: The worldwide installments area is mistake inclined, expensive, and open to illegal tax avoidance. It takes days if not longer for cash to cross the world. The block chain is as of now furnishing arrangements with settlement

3. Organizations, for example, Align Commerce and Bit spark that offer end-to-end block chain controlled settlement administrations. In 2004, Santander wound up one of the primary banks to combine block chain to an installments application, empowering clients to make universal installments 24 hours per day, while clearing the following day.

ii) Smart property: An unmistakable or elusive property, the decentralized record additionally turns into a framework for recording and overseeing property

Rights and in addition empowering the savvy contracts to be copied if records or the brilliant key is lost. Influencing property to keen reductions your dangers of running into misrepresentation, intervention Expenses, and sketchy business circumstances. In the meantime, it expands trust and productivity.

i. Cars/smartphone: Crude types of savvy property exist. Your auto scratch, for example, might be furnished with an immobilizer, where the auto must be initiated once you tap the correct Convention on the key. Your cell phone too will just capacity once you write in the correct Pin code. Both work on cryptography to secure your proprietorship. The issue with crude types of savvy property is that the key is normally held in a physical Compartment, for example, the auto key or SIM card, and can't be effectively exchanged or replicated. The block chain record takes care of this issue by enabling block chain mineworkers to Supplant and repeat a lost convention. 


\section{iii) Smart contracts}

Smart contracts are advanced which are implanted with an if-this-then-that (IFTTT) code, which gives themself-execution. In actuality, a go-between guarantees that all gatherings complete on terms. The Block chain forgoes the requirement for outsiders, as well as guarantees that all record members know

The agreement subtle elements and that legally binding terms execute naturally once conditions are met. You can utilize keen contracts for all kind of circumstances, for example, monetary subordinates, protection premiums, property law, and group financing understandings, among others.

1. Music: Enter issues in the music business incorporate proprietorship rights, sovereignty dispersion, and straight forwardness. The advanced music industry centers around adapting preparations, while possession rights are regularly ignored. The block chain and keen contracts innovation can circuit this issue by making a thorough and exact decentralized database of music rights. In the meantime, the record and give straightforward transmission of craftsman eminences and continuous conveyances to all required with the marks. Players would be paid with computerized money as per the predetermined terms of the agreement.

2. Identity: In any case, online organizations thoroughly understand us. A few organizations whom we buy from offer our personality points of interest to sponsors who send you their advertisements. The block chain block this for making a secured information point where you just wanted the individuals to see the data at a certain time.

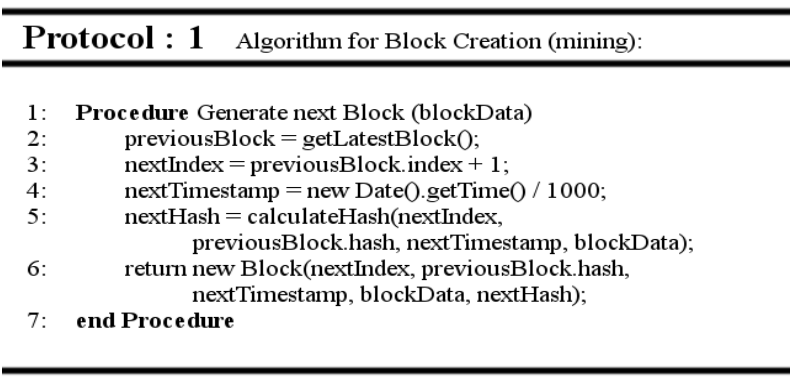

\begin{tabular}{|c|c|}
\hline & tocol : 2 Validating the integrity of blocks \\
\hline $\begin{array}{l}\text { 1: } \\
2: \\
3: \\
4: \\
5: \\
6: \\
7: \\
8:\end{array}$ & $\begin{array}{l}\text { ValidNewBlock }=\text { (newBlock, previousBlock) } \\
\text { if previousBlock.index }+1 \text { not equal newBlock.index: } \\
\text { return false } / / \text { invalid index } \\
\text { else if previousBlock.hash not equal newBlock.previousHash } \\
\text { retum false //invalid previoushash } \\
\text { else if calculateHashFornewBlock not equal newBlock.hash } \\
\text { return false//invalid hash } \\
\text { return true; }\end{array}$ \\
\hline
\end{tabular}

\section{Architecture}

The justifying peers runs on BFT consensus protocol for producing duplicate state machine that is going to acquire.

There are 3 types of transactions as per operations:

Deploy transaction: It pats a chain code which is written as Go as a parameter; the chain code introduced on the peers and it access request.

Invoke transaction: Request the proceeding of the chain code which is installed by a deploy transaction. The Arguments are definite as sort of a transaction. The chain code will execute the transaction, that will perused and compose sections clinched alongside its state Appropriately What's more show that it will be accomplished or neglected.

Query Transaction: Returns an entrance specifically starting with perusing the peer's constant state, this might not guarantee linearizability. Each chain code might define its own constant sections in the state. The piece chain's hash chain will be registered through the executed transactions and the coming about constant state. Acceptance about transactions happens through the replicated execution of the chain code What's more provided for those shortcoming suspicion underlying BFT consensus, i.e., That "around those $n$ accepting companions at most $\mathrm{f}<\mathrm{n} / 3$ might "lie" and carry on arbitrarily, at constantly on others execute those chain code effectively. When executed ahead highest priority on PBFT consensus, it is critical that chain code transactions are deterministic, Overall the state of the companions might veer. a secluded answer for filter crazy non-deterministic transactions that need aid certifiably wandering will be accessible and need been executed in the sifter protocol. Enrollment Around the accepting hubs running BFT agreement will be at present static and the setup obliges manual mediation. Backing to rapidly evolving those set from claiming hubs running agreement is wanted for A future rendition. As the fabric executes A permissioned ledger, it holds A security framework to verification \& commission. It helps enlistment and transaction commission through public-key certificates, also confidentiality to chain code understood through in-band encryption. Even more precisely, to interfacing of the system each companion needs on acquire an enlistment certificate from an enlistment ca that is and only those enrollment benefits. It Sanctions a companion on unite with those organize Also to obtain transaction certificates, which need aid necessary on submit transactions.

Transaction certificates are issued Toward a transaction What's more backing pseudonymous commission to the companions Submitting transactions, means Different transaction certificate issued of the same companion can't a chance to be joined with one another. Confidentiality to chain codes What's more state is Gave through symmetric-key encryption from claiming transactions Furthermore states for a piece chain specific way that is accessible should every bit associates for an enlistment certificate to those block chain. Continuing the encryption instruments against additional fine-grained confidentiality to transactions Furthermore state sections will be arranged to a future adaptation.

\section{Hyper ledger fabric}

It may be an execution of a imparted record stage to running keen contracts, leveraging commonplace Also turned out technologies, for An secluded structural engineering permitting pluggable use of diverse capacities. It may be a standout amongst numerous ventures right now in brooding under those hyper record one task. Those conveyed record protocol of the fabric may be run by associates. Those fabric recognizes between two sorts of peers a accepting companion will be a hub on the organize answerable for running consensus, accepting transactions, and keeping up the record. On the other hand, a non-approving companion may be a hub that capacities Likewise anenvoy will associate customers on accepting associates. A non-validating companion doesn't execute transactions, yet it might check them.

Some of the key features of fabric are:

- A permitted block chain with actua lintegrity.

- Runs impulsive sensible contracts (called chain code) enforced in Go (golang.org): User-defined chain code was self-enclosed in a very dock-walloper container; -System chain code can run within the same method as peer

- Consensus protocol is plowable, presently associate degree implementation of Byzantine fault-tolerant accord exploitation the PBFT protocol [4] are supported, a paradigm of SIEVE [3] to deal with the non-deterministic chain code are obtainable, and a protocol stub (named NOOPS) serves for development on one node.

- $\quad$ Security support through certificate authorities (CAs) for TLS certificates, enrollment certificates, and dealing certificates.

- $\quad$ persistency state utilizing a key-value store interface, 
sponsored Toward Rocks db (rocksdb. Org);

- $\quad$ an occurrence framework that supports pre-defined and custom events;

- A customer SDK (Node.js) with interface with those fabric.

- Help essential REST APIs and CLIs.

\section{Figures}

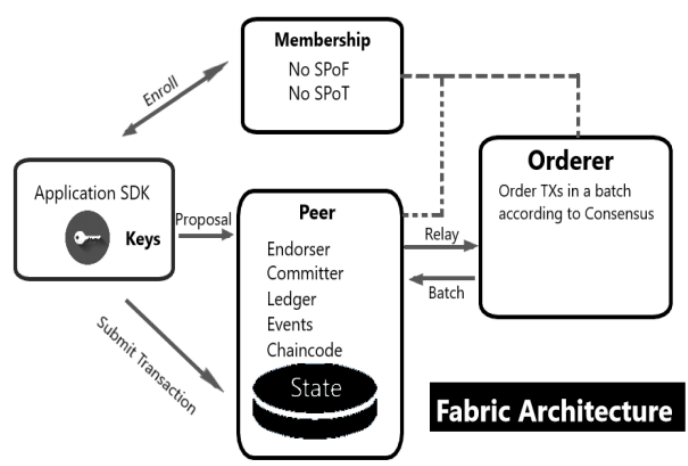

Figure 1: Architecture of hyperledger fabric

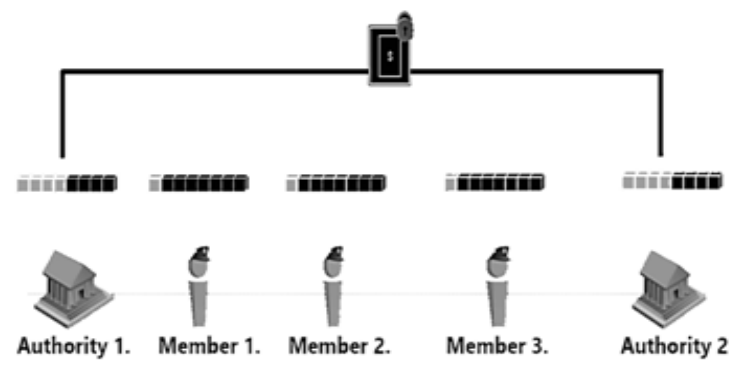

Figure 2: Business model block chain example

\section{Results}
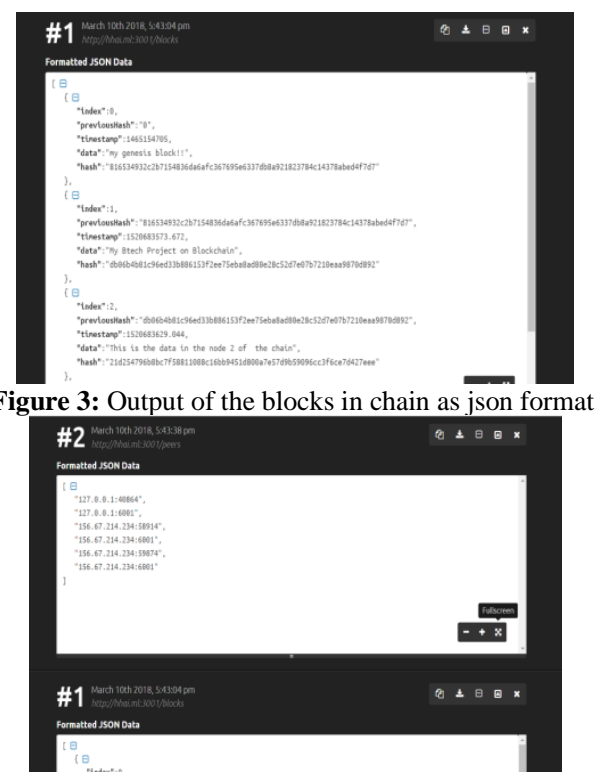

Figure 4: Output of the peers connected to the chain

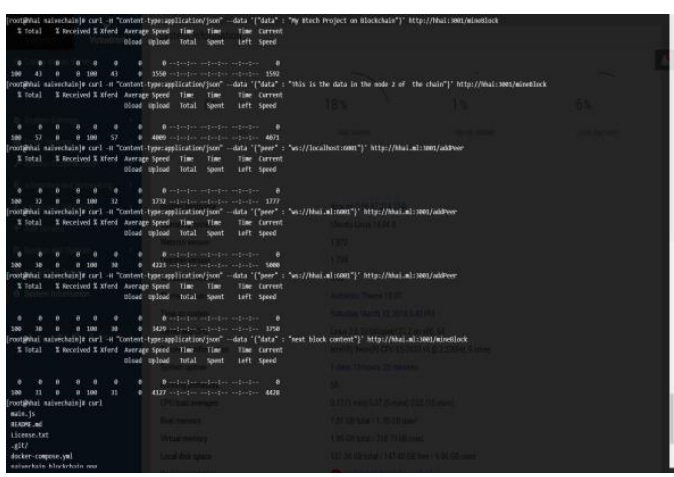

Figure 5: proof of work implementation of the chain

\section{References}

[1] Cachin C, "Architecture of the Hyperledger blockchain fabric", Workshop on Distributed Cryptocurrencies and Consensus Ledgers, (2016).

[2] https://hyperledger.org/

[3] Valenta M \& Sandner P, "Comparison of Ethereum, Hyperledger Fabric and Corda", FSBC Working Paper, (2017).

[4] Vukolic M, "The quest for scalable block chain fabric", Workshop on Open Problems in Network Security, (2015).

[5] Gervais A, Karame GO, Wüst K \& Glykantzis V, "On the security and performance of proof of work block chains", Proceedings of the ACM SIGSAC Conference on Computer and Communications Security, (2016). 